\title{
NOTAS
}

\section{Pulcre cogitandum: analogías en la argumentación estética}

\author{
MARIANELA CALLEJA \\ Universidad Nacional del Sur
}

Resumen: En sus recientes libros, Cuestiones de fundamento (2014) y Reglas y diálogos: una discusión lógica (2016), Jorge Roetti trata sobre las analogías como ejemplo de fundamentación "insuficiente". El propósito de este trabajo es desarrollar este planteo, llevándolo desde una posición leibniciana hacia una baumgartiana: llegando a abordar las analogías positivamente, como recurso ampliativo de conocimiento, atendiendo especialmente a su relevancia en la metateoría o fundamentación de tesis en estética. Nos detendremos en el caso especial de las analogías cualitativas, cuyo sentido de la semejanza, si bien es acotable, no puede determinarse por un procedimiento exacto.

Palabras clave: conocimiento estético, analogías cualitativas, tipología de las analogías, semejanza, herencia leibniciana. 


\title{
Pulcre cogitandum: Analogies in Aesthetic Argumentation
}

\begin{abstract}
In his recent books, Cuestiones de fundamento (2014) and Reglas y diálogos: una discusión lógica (2016), Jorge Roetti deals with analogies as an example of "insufficient" reason. The purpose of this paper is to develop this approach, taking it from a Leibnician position to a Baumgartian one: coming to approach the analogies positively, as an expansive resource of knowledge, paying particular attention to its relevance in the metatheory or foundation of thesis in aesthetics. We will dwell on the special case of qualitative analogies, whose sense of similarity, although it can be limited, cannot be determined by an exact procedure.
\end{abstract}

Key-words: aesthetic knowledge, qualitative analogies, typology of analogies, similarity, Leibnizian heritage.

\section{Analogía y fundamentación insuficiente}

Z términos leibnicianos, Jorge Roetti argumenta que la funa aquellos enunciados para los que toda objeción posible no es respondida de manera suficiente o perfecta. Una tesis suficientemente fundada, por el contrario, satisface estas condiciones: todas las hipótesis son verdaderas y la regla de paso entre sus premisas conserva la verdad (Roetti 2014: 145). Por lo tanto, un enunciado será fundable insuficientemente en los siguientes casos:

(1) Cuando, ante un cuestionamiento, el orador pueda presentar al menos una entidad (o una colección de entidades) externa(s) al lenguaje del enunciado, que baste para persuadir a la audiencia o los cuestionadores (esto es lo que corresponde a la teoría de la correspondencia y remite a la noción de "verosimilitud" de la misma), o bien

(2) Cuando puede ofrecer a la audiencia o los cuestionadores un argumento sobre alguna forma de presunta compatibilidad del enunciado con un sistema de otros enunciados previamente admitidos por esta audiencia, aunque dicha compatibilidad no suponga necesariamente una demostración (esto remite a una versión coherentista de la "verosimilitud" del enunciado) (Roetti 2014: 56-57).

La fundamentación insuficiente no es atributo peculiar de las argumentaciones por analogía, sino que ella incluye también a otras formas de argumentación como la inducción empírica, la abducción, las correlaciones y las inferencias probabilísticas. 
Como ya se definió arriba, las analogías son imperfectas en tanto puede haber, en los silogismos en que ellas se expresan, una regla de paso problemática o bien los enunciados pueden contener concepciones problemáticas, o ambas situaciones. Las tesis insuficientemente fundadas se caracterizan por un fundamento que, o bien ha superado mínimamente objeciones pero no todas las objeciones posibles, o su regla de paso es imperfecta, es decir, no conserva el grado de fundamento de la hipótesis menos fundada, o ambas cosas (para su definición formal, véase Roetti 2014: 145-146).

Desde un punto de vista histórico, Aristóteles dividió (Top. I, 1: 100a25-101a4) entre inducción epistémica y dialéctica. La inducción epistémica "justifica enunciados universales a partir de un (solo) ejemplo particular". Así, este tipo de inducción se corresponde con una argumentación con demostración suficiente en términos de la teoría de la fundamentación de Roetti. Este tipo de demostraciones son frecuentes en ciencias constructivas o simbólicas como la matemática o la lógica y en las protociencias de ciencias empíricas como la protofísica, que "consiste en la presentación de ejemplos, forzosamente individuales, pero que son generados mediante una regla, esquema o norma de construcción, que es ella misma universal" (Roetti 2016: 391). Los ejemplos mencionados son: la construcción de los esquemas de la imaginación temporal y espacial en Kant, la construcción en el tiempo del intuicionismo matemático, entre otras construcciones de objetos teóricos en general. Por su parte, la inducción dialéctica solo "justifica enunciados generales a partir de una pluralidad de casos particulares" (Roetti 2016:389).

Roetti divide entre silogismos dialécticos de fundamentación imperfecta (sd1) y (sd2). Sd1 se caracteriza porque una de sus premisas está insuficientemente fundada y la conclusión está fundada mediante una regla falible (Roetti 2014: 156). Sd2 se caracteriza porque todos sus enunciados están suficientemente fundados pero su regla de paso es falible (Roetti 2014: 157).

Las analogías o proporciones surgieron como "instrumento metódico para la aclaración de estados de cosas de diferentes dominios de objetos" (Roetti 2016: 413-424). Las analogías matemáticas "8:4=4:2" o ternas del tipo $\langle 6,4,3>$, donde 6 excede a 4 por un tercio y, a su vez, 3 es excedido por un tercio por 4 , permiten calcular el término medio objetivamente. Las analogías en otros dominios, como en la ética aristotélica, nos exige la búsqueda de un término medio entre exceso y defecto, sin embargo no calculable. "La justicia, por ejemplo, es una relación al menos cuadripartita entre dos personas y sus fines o intereses (obras, acciones, cosas, etc.)" (Roetti 2016: 416).

Pablo Oyarzún, en el prólogo a su traducción de la Crítica de la facultad de juzgar, trata el lugar del juicio reflexionante y su rol en el ejercicio de discernimiento en problemas de indeterminación. Oyarzún, como Roetti, cita en primera instancia el caso de la jurisprudencia, es decir, la indeterminación 
como un problema primeramente ético (Oyarzún 1992: 13). El tema de las analogías como caso límite en cuestiones de fundamento se propone desde esta perspectiva avanzar sobre una fenomenología en diversas áreas de la filosofia: analogías en metafisica, ética, filosofia política, económica, estética (véase Roetti y Moro 2016). Aquí solo nos concentraremos en el último grupo de aplicación y pondremos énfasis para ello en algunas cuestiones del debate contemporáneo.

Avanzaremos hacia una clasificación de las analogías, partiremos de su interpretación matemática hasta llegar a los límites difusos de la metáfora:

- Analogías cuantitativas: existe un procedimiento exacto para calcular.

- Analogías cualitativas: no son calculables de manera exacta, pero todavía sus semejanzas son acotables.

Roetti detiene su análisis aquí, pero todavía podemos añadir:

- Metáforas: no está acotado el sentido de la semejanza, sus semejanzas son abiertas e indeterminadas.

Si nos detenemos en la clasificación de las analogías cualitativas, que son las que aquí nos interesan especialmente, todavía podremos dividir entre:

- Analogías estructurales: relaciones entre sistemas de objetos (Roetti 2016: 420; tomadas de Kuno Lorenz). Estas fueron particularmente expresadas en términos de analogías constitutivas o constructivas, pero son aplicables a las analogías de tipo cualitativas. Ejemplo en la lógica clásica:

Sean dos especies E1 y E2 de un género G y sea una cualidad Q para la cual valen que: (1) 'Todos los E1 son Q'y (2) 'Todos los E2 son Q', entonces si (3) 'Todos los E1 son P', se puede concluir por analogía: (4) 'Todos los E2 son P', si suponemos que (5) 'Todos los Q son P'. - Analogías funcionales: cuando sistemas de objetos son adecuados para una misma tarea aunque sean diferentes las especies de sus elementos e incluso no sean estructuralmente análogos (Roetti 2016: 422; tomadas de Christian Thiel).

Nos movemos un momento de las clasificaciones en el ámbito de la lógica a la situación de la analogía en la filosofía del lenguaje. Ludwig Wittgenstein indagó en este tema. El lenguaje es por un lado una pintura, una representación, hasta llegar a la idea de lenguaje como analogía ambigua, por lo complicado y variado. Carla Cordua lo expresa claramente:

El paso a la segunda época de su pensamiento no está señalado por el abandono de la idea del lenguaje como representación del mundo sino más bien por el incesante cuestionamiento y progresiva complicación de dicha 
idea. [...] ahora el carácter representativo de la realidad que el lenguaje tiene no será, por un lado, sino una analogía ambigua, por otro, una comparación para clarificar el carácter del lenguaje entre otras comparaciones metódicas. (Cordua 1996: 193)

Nos remitimos a Wittgenstein, especialmente al segundo período de su filosofia, porque atiende al método comparativo como distintivo del quehacer filosófico: la perpetua búsqueda de diferencias y parecidos y de claridad tranquilizadora en la confusión. Wittgenstein nos advierte asimismo del peligro de la comparación de todo con todo que conlleva este método.

Sin embargo, la función de la analogía es iluminadora, describe usos simbólicos que no están dados como los fenómenos; es preciso distinguir entonces entre analogía sobre fenómenos y analogías del lenguaje o sobre el lenguaje. Lo cierto es que las analogías para Wittgenstein no prueban nada. Según Roetti, no lo pretenden.

Las analogías son corrientes, filosóficas o aún artísticas. Una importante analogía a la que se dedica es la del lenguaje como instrumento. Las palabras, como los instrumentos, poseen diversas utilidades:

Piensa en las herramientas en una caja de herramientas: hay allí un martillo, unas tenazas, un serrucho, un destornillador, un metro, una lata para cola, cola, clavos y tornillos. Tan diversas como las funciones de estos objetos son las funciones de las palabras (Wittgenstein 1953: \$11).

Esta analogía nos revela las cosas que hacemos con palabras, que Cordua explicita:“[...] revelar intenciones, avisar novedades, expresar necesidades, comunicar conocimiento, narrar recuerdos, contar sueños, señalar direcciones, hacer promesas [...]" (Cordua 1996: 200).

La dimensión del uso de analogías en filosofía se ejemplifica en la obra de Wittgenstein por lo fecundas de sus comparaciones y por la repercusión en toda la investigación filosófica posterior:

Por una parte arroja luz sobre el lenguaje, nuestra relación con él, el lugar que ocupa en nuestra vida y abre la perspectiva de la concepción wittgensteiniana del significado como uso. Por otro lado, hace un aporte fecundo a la investigación de las palabras que designan conceptos psicológicos [...] El examen de los múltiples aspectos del ver, por ejemplo, comprende el distingo entre ver y mirar (Cordua 1996: 208).

Una segunda aproximación a figuras análogas del lenguaje la elabora Wittgenstein sobre los límites que esta primera analogía de "lenguaje-he- 
rramienta" le impone. A diferencia de los instrumentos, las palabras parecieran tener usos más variados; otro aspecto que las hace diferente es que algunas parecieran no "servir para nada". En esta nueva concepción entran los chistes, los juegos de palabras, los gritos o exclamaciones expresivos, lo cantado o murmurado, todas situaciones que Wittgenstein ubica en la figura análoga del juego (véase Cordua 1996: 210).

\section{Analogía y conocimiento estético}

Os propusimos al inicio abocarnos a las analogías en el dominio
estético. Si nos remontamos ya a los comienzos de la disciplina, según Alexander G. Baumgarten en su Metafísica, la perspicacia (Einsicht), la agudeza y el discernimiento implican una poderosa facultad de sentir y concebir analogías:

$\int 572$. Percibo identidades y diferencias entre las cosas. Luego, tengo la facultad de percibir identidades y diferencias entre las cosas, $\$ 216$. La primera facultad sería mínima, si bastara solo para representar de manera muy débil, respecto de dos percepciones poderosísimas, extremadamente semejantes, una identidad mínima en medio de percepciones heterogéneas extremadamente débiles, asociadas o anteriores. Por ende, tanto mayor es esta facultad cuanto más numerosas, cuanto menos notorias, cuanto más diversas, cuanto más frecuentes y mayores son tanto las identidades como las correspondencias y las igualdades de medida o PROPORCIONES, las similitudes; cuanto más poderosas en medio de percepciones heterogéneas asociadas y anteriores, cuanto más claramente son percibidas $\mathbb{2 1 9}$. El hábito de observar identidades entre las cosas es el INGENIO EN SENTIDO ESTRICTO. (Baumgarten 1739: 19-20)

Por otra parte, el "análogo de la razón” o conocimiento sensible, facultad propia del objeto estético en Baumgarten, nos remite directamente a su particular lectura de Leibniz:

Lo característico de Baumgarten es que para él el pensar algo no-claramente significa representar algo expresivamente, esto, no se trata de un no-conocimiento, sino de un conocimiento distinto al del lógico-abstractivo. (Soto Bruna 1987: 185)

Comenzamos con la definición de razón insuficiente de Roetti, de herencia leibniciana, para profundizar hacia una noción de razón ampliativa, creativa, que se inicia en la estética de Baumgarten: 
Esta representación sensible, donde se sitúa la captación del objeto estético, es para Baumgarten un analogum rationis, que no es solo la sensibilidad de Leibniz o de Wolff sino que contiene un mayor valor gnoseológico y psicológico [...] Mientras que para Leibniz la intuición sensible era un "todavía no” del pensamiento, para Baumgarten es el análogo de la razón, con una función propia, a saber, representar el conjunto de la multiplicidad de los objetos sensibles. (Soto Bruna 1987: 185)

Baumgarten hereda a su vez que amplía la noción de conocimiento de Leibniz. El fin de todo conocimiento para Leibniz es la perfección:

Pero la unidad en la multiplicidad no es otra cosa que la coincidencia, y porque una cosa coincide más con esta que con aquella, fluye el orden del que procede toda belleza, y la belleza despierta el amor. Así se ven felicidad, placer, amor, perfección, ser, fuerza, libertad, coincidencia, orden y belleza unidos entre sí, cosa en la que pocos reparan (EF,VII).

Esa "dispositio naturalis animae totius ad pulcre cogitandum" o "arte del pensar bellamente” según Baumgarten (1752-1758a, \$28 y 1752-158b, \$1: 31 , proporcionaría el perfeccionamiento del conocimiento sensible.

\section{Analogías y argumentación en estética}

T a teoría estética contemporánea nos proporciona diversos

Lejemplos de fundamentación de sus tesis a través del recurso a la analogía. Es conveniente aclarar que nos detendremos en un aspecto de la metateoría, no el arte, sino el discurso sobre el arte.

Una posible clasificación de analogías en la fundamentación estética contemporánea podría incluir al menos los siguientes tipos principales, aun sin ser exhaustiva:

- Perceptuales: semejanza entre universales perceptivos.

En el ámbito de la filosofía de la música, según Jennifer Robinson escuchamos movimiento en la música, cuando sin embargo nada se mueve, en el sentido de la traslación de objetos, debido a la actividad del sistema motor. Lo mismo ocurre con las emociones, que no están la música, sino que se despiertan en nosotros por un proceso de mímica. El paso defendible, aunque débilmente (ya no diremos insuficientemente), es el que se da en la analogía del contagio emocional entre expresiones faciales y corporales entre personas, y las expresiones y movimientos que activa la música: 
La música es parecida a los humanos y puede sonar y mover como humano pero no es un humano [...] Más importante, las emociones empáticas se sienten por o en nombre de otra persona, pero escuchando música triste o alegre no hay persona por la que sintamos esa tristeza o alegría. Los sentimientos pertenecen a nosotros mismos (Robinson 2016: 8; traducción de la autora).

Un segundo aspecto que revela la debilidad del argumento mediante analogías es la capacidad que tiene de dejar sin acotar ciertas interpretaciones opuestas: con respecto al argumento sobre la mímica emocional, los formalistas defenderán asimismo que solo detectamos movimientos y expresiones emocionales en la música sin en verdad conmovernos o sentir algo nosotros mismos (véase Robinson 2016: 9).

Nos hallamos pues ante un caso de analogía cualitativa-estructural; el silogismo al que podemos reducir este argumento es del tipo Sd1. La analogía entre emociones en la música-emociones humanas se hallaría débilmente fundada. Al menos sería falible el enunciado sobre las emociones en la música, tanto como las conclusiones son falibles.

- Cognitivas: semejanza entre operaciones epistémicas y apreciación estética.

En el ámbito de la estética cognitiva, un ejemplo de analogía estructural es el que desarrolla Jérôme Dokic en su intento por dar una explicación de la experiencia estética como una variedad de sentimiento epistémico, específicamente como "la reflexión fenomenológica de la fluidez del procesamiento" (Dokic 2016: 48). Esta fluidez responde a la facilidad de procesamiento, bajo esfuerzo y alta velocidad; mientras la no fluidez, por el contrario, al carácter complejo, nuevo o no familiar de ciertos estímulos.

Los problemas o debilidades que surgen de esta analogía es concretamente que "los artistas seleccionan a menudo obras de arte que provocan no fluidez de procesamiento, al exhibir complejidad, no familiaridad, extrañamiento, no armonía, desbalance, indeterminación, incertidumbre" (Dokic 2016: 51).

Por lo tanto, la analogía fluidez-placer o fluidez-goce estético y su opuesta, no fluidez-displacer, es débil por lo anteriormente expuesto; de modo que nuevamente nos hallamos ante una ampliación de nuestra comprensión acerca de lo que involucra la experiencia estética, pero que es débilmente fundable. Ni una objeción al menos puede ser defendida.

En los términos desarrollados, se trataría de un caso de analogía estructural, cuyo silogismo es de tipo Sd1, donde ambas premisas son falibles: placer-familiaridad/goce estético-familiaridad. Por tanto, asimismo su conclusión es falible.

- Evaluativas: semejanza por adecuarse o reproducir un estándar o modelo.

En el ámbito de la filosofia de la crítica, Jean-Marie Schaeffer de- 
sarrolló un programa descriptivista (véase Ibarlucía 2016; Schaeffer 1996, 2000, 2013a). Este consiste en dar preponderancia a los aspectos técnicos y la adecuación a modelos preestablecidos (juicios teleológicos) como tarea primordial de la crítica, dejando de lado apreciaciones subjetivas (juicio estético). Schaeffer fundará su punto de vista en la analogía obra particular-procedimiento técnico objetivable.

Sin embargo, Ricardo Ibarlucía defiende un modelo superador de las posiciones descriptivista y evaluacionista de la crítica de arte actual, mostrando la falencia de la argumentación que solo puede defenderse de al menos una objeción pero que está en situación de equiparamiento respecto de otras argumentaciones, aunque de sentido opuesto, pero similares en cuanto a su fuerza explicativa. Según la primera, como señalamos, los juicios de la crítica incluyen descripciones de las propiedades objetuales, son juicios técnicos; según la segunda posición, defendida por Noël Carroll (2009), la crítica debería atender a las preferencias subjetivas o juicios de gusto. Para Ibarlucía, estos aspectos pueden conjugarse:"el juicio crítico [...] expone bajo la forma de predicados de valor las propiedades objetuales que constituyen la causa de la apreciación" (Ibarlucía 2016: 222).

Aquí nos encontramos ante un caso de analogía funcional entre modelo standard objetivo-obra particular o reproducción del modelo. Se trata de un $\mathrm{Sd} 1$ en tanto sus enunciados o premisas no son demostrables (en qué sentido es este modelo creado/subjetivo o descubierto/objetivo) y el paso de una a otra tampoco: la proporcionalidad cualitativa hace indemostrable la relación, porque es indeterminable.

- Heurísticas: semejanza entre órdenes diversos, biológicos y artísticos.

Una aproximación a la estética desde la filosofia natural, como la del ya antes citado Jean-Marie Schaeffer, basa su argumentación en una homología o, en nuestros términos, analogía estructural. Schaeffer habla en términos de "arquitectura" (del nido de las aves que construyen emparrados), "decoración" (ya que estos nidos se pintan y se reacondicionan con flores frescas, por ejemplo), "canto" (que produce el macho en su interior como despliegue para seducción de la hembra), "observación" (de la hembra que atiende al espectáculo), y "elección de preferencias" (la hembra podrá elegir o rechazar la iniciativa del macho). La analogía entonces estaría dada entre la creación y apreciación del espectáculo de las aves de emparrado, con nuestra creación y apreciación estéticas.

Esta analogía pronto trae dificultades, como todas las analogías anteriormente expuestas. Una principal es su interpretación en términos funcionales, llevando la propuesta a entender el arte en términos de su servicio a la selección sexual. La analogía se mantiene porque dos situaciones especialmente la sustentan: que el macho elabore el emparrado no con el fin con el 
que, por ejemplo, elabora la hembra el suyo para sus pichones, sino con un propósito exhibitivo y, segundo, que haya un tiempo del cortejo distinto del tiempo del apareamiento en sí mismo.

Dos situaciones se añaden a la argumentación por analogías: la señalización costosa o empeño poco económico de las tareas de construcción y exhibición del macho, y las de inversión cognitiva por parte de la hembra que, tanto como el macho, desplaza reacciones comportamentales inmediatas y es capaz de sincronizar con la propuesta del espectáculo.

La analogía que aquí tratamos tendrá el propósito de inventar una hipótesis que luego deberá ser finamente elaborada, ya que esta analogía de por sí no explicaría absolutamente que las aves experimenten una experiencia estética al modo en que los humanos la experimentamos. Sin embargo, la importancia heurística que reporta es la de aumentar nuestra comprensión de los procesos perceptuales y cognitivos implicados en la apreciación: niveles descendentes de atención, estados cognitivos divergentes y categorización retardada (Schaeffer 2013b: 16-24).

Nos encontramos ante un caso de analogía funcional entre el despliegue-cortejo de las aves y los procesos atencionales durante la apreciación estética.Y desde un punto de vista de la argumentación, ante un $\mathrm{Sd} 2$, porque sus enunciados están demostrados en campos diversos pero su paso de uno a otro trae problemas en tanto la relación no es ni isomórfica ni biunívoca; el isomorfismo requiere una preservación o invariancia estructural y los sistemas de relaciones de un campo concuerdan solo parcialmente con los del otro.

\section{Conclusiones}

T emos definido lo que consideramos fundamentación de una tesis

1 por contraposición a su demostración, y los problemas relativos a su fundamentación en dominios problemáticos. Nos hemos ocupado al final de las analogías en estética y de una breve tipología. Podríamos avanzar todavía y hallar, en esta tipología, diferentes niveles de análisis, relativos a:

- Experiencia y apreciación estética: analogías perceptuales y cognitivas.

- Evaluación y descripción/investigación estética: analogías evaluativas y heurísticas.

Las analogías como recurso en la argumentación estética no se relacionan estrictamente ni a un razonamiento probable, ni a un recurso literario que impacte por su efecto vívido en un discurso. Tienen relaciones más directas con los usos escolásticos sobre proporción, aunque estas servían 
para explicar la relación entre un ser supremo y los demás seres (Ferrater Mora 1994: 158-160).

Una teoría de la razón ampliada, en este contexto, que admita como legítimas las fundamentaciones demostrables tanto como las simplemente fundables, nos permite entender las analogías por ejemplo como medios para proponer la superación de tesis reduccionistas (véase Calleja 2016: 250251), en tanto son argumentos que añaden otras dimensiones a nuestra comprensión, en este caso, sobre los objetos estéticos o sobre teorías en torno al objeto estético. Estos no son ni totalmente acotables ni exactos, sin embargo son regulables por medio del alcance explicativo que otorgan.

Finalmente, las analogías como recurso para la fundamentación poseen la mayor de las debilidades y sin embargo, su fuerza radica en la creatividad que despliegan sus argumentos, sin la cual ningún conocimiento nuevo sería posible. Como conclusión, nos quedamos con dos situaciones: si bien las analogías no prueban definitivamente nada, constituyen un recurso indispensable para la invención, revisión y ampliación del conocimiento en general, en cuyo caso el conocimiento estético merece un lugar privilegiado de acceso.

\section{BIBLIOGRAFÍA}

Aristóteles (Top.), The Complete Works of Aristotle I:Topics, edición de J. Barnes (Princeton: Princeton University Press, 1991).

Baumgarten, A. G. (1739), Metafísica, en A. G. Baumgarten (2014), Estética breve, prólogo, selección, traducción y notas de R. Ibarlucía (Buenos Aires: CIF, Excursus, 13-14).

Baumgarten, A. G. (1750-1758a), Estética, en A. G. Baumgarten (2014), Estética breve, prólogo, selección, traducción y notas de R. Ibarlucía (Buenos Aires: CIF, Excursus, 31-40).

Baumgarten, A. G. (1750-1758b), Aesthetica/Ästhetik, edición de D. Mirbach, 2 vols. (Hamburg: Felix Meiner, 2007).

Baumgarten, A. G. (2014), Estética breve, prólogo, selección, traducción y notas de R. Ibarlucía (Buenos Aires: CIF, Excursus).

Calleja, M. (2016), "La explicación por analogía y el tiempo musical”, en J. Roetti (2016), Reglas y diálogos: una discusión lógica (Buenos Aires: Academia Nacional de Ciencias de Buenos Aires, Centro de Estudios Filosóficos Eugenio Pucciarelli, 237-253).

Carroll, N. (2009), On Criticism (New York and London: Routledge).

Cordua, C. (1996), "Wittgenstein: análogos del lenguaje", ARETÉ: Revista de filosofía, 8 (2): 191-213. 
Dokic, J. (2016), “¿La experiencia estética como un sentimiento metacognitivo?: una visión de aspecto dual”, Boletín de Estética, 35: 35-65.

Ferrater Mora, J. (1994), Diccionario de Filosofía (Barcelona: Ariel).

Ibarlucía, R. "Descripción y evaluación: algunas observaciones sobre el discurso de la crítica”, en D. Pérez y R. Ibarlucía (2016) (comps.), Hechos y valores en filosofía teórica, filosofía práctica y filosofía del arte (Buenos Aires: CIF/SADAF, 213-224).

Kant, E. (1790) Crítica de la facultad de juzgar, traducción, introducción y notas de P. Oyarzún (Venezuela: Monte Ávila, 1992).

Leibniz, G.W. (EF), Escritos filosóficos VII. Escritos alemanes de la sabiduría: 4. De la sabiduría, edición de E. de Olaso y R. Torretti (Buenos Aires: Charcas, 1992).

Oyarzún, P. (1992), “Introducción del traductor”, en E. Kant (1790), Crítica de la facultad de juzgar (Venezuela: Monte Ávila, 1992, 7-17).

Pérez, D. e Ibarlucía, R. (2016) (comps.), Hechos y valores en filosofía teórica, filosofía práctica y filosofía del arte (Buenos Aires: CIF/SADAF).

Roetti, J. (2014), Cuestiones de fundamento (Buenos Aires: Academia Nacional de Ciencias de Buenos Aires, Centro de Estudios Filosóficos Eugenio Pucciarelli).

Roetti, J. (2016), Reglas y diálogos: una discusión lógica (Buenos Aires: Academia Nacional de Ciencias de Buenos Aires, Centro de Estudios Filosóficos Eugenio Pucciarelli).

Roetti, J. y Moro, R. (2016), El fundamento y sus límites: algunos problemas de fundamentación en ciencia y filosofía (Londres: College Publications).

Robinson, J. (2016), "Empathy and Music", presentación oral para el Workshop "Segunda persona y emociones" (Buenos Aires: SADAF).

Schaeffer, J.-M. (1996), Les célibataires de l'art: pour une esthétique sans mythes (París: Gallimard).

Schaeffer, J.-M. (2000), Adieu à l'esthétique (París: PUF).

Schaeffer, J.-M. (2013a), Pequeña ecología de los estudios literarios: ¿por qué y cómo estudiar la literatura?, traducción de L. Folica (Buenos Aires: Fondo de Cultura Económica).

Schaeffer, J.-M. (2013b), "Experiencia estética: placer y conocimiento", Boletín de Estética, 25: 5-24.

Soto Bruna, M. J. (1987), "La Aesthetica de Baumgarten y sus antecedentes leibnicianos”, Anuario Filosófico, 20: 181-190.

Wittgenstein, L. (1953), Philosophical Investigations (Londres: Blackwell).

Recibido: 02-03-2017; aceptado: 11-11-2018 